\title{
Adaptive protocols based on predictions from a mechanistic model of the effect of IL7 on CD4 counts
}

\author{
Laura Villain*1,2,3 | Daniel Commenges ${ }^{1,2,3}$ | Chloé Pasin ${ }^{1,2,3}$ | Mélanie Prague Ch,3 $^{1,2,3 o d o l p h e}$ \\ Thiébaut ${ }^{1,2,3}$
}

${ }^{1}$ Univ. Bordeaux, Inserm, Bordeaux Population Health Research Center, team SISTM, UMR 1219146 rue Léo Saignat, Bordeaux France

${ }^{2}$ INRIA Bordeaux Sud Ouest, Talence, France

${ }^{3}$ Vaccine Research Institute, VRI, Hôpital Henri Mordor, 51 avenue du Maréchal de Lattre de Tassigny, Créteil, France

\section{Correspondence}

*Rodolphe Thiebaut Email:

rodolphe.thiebaut@u-bordeaux.fr

\section{Present Address}

INSERM U1219, ISPED, Univ. Bordeaux, 146 Rue Leo Saignat, 33076 Bordeaux, France

\begin{abstract}
Summary
In HIV infected patients, antiretroviral therapy suppresses the viral replication, which is followed in most patients by a restoration of CD4+ T cells pool. For patients who fail to do so, repeated injections of exogenous interleukin 7 (IL7) are experimented. IL7 is a cytokine that is involved in the T cell homeostasis and the INSPIRE study has shown that injections of IL7 induced a proliferation of CD4+ T cells. Phase I/II INSPIRE 2 and 3 studies have evaluated a protocol in which a first cycle of three IL7 injections is followed by a new cycle at each visit when the patient has less than 550 CD 4 cells $/ \mu L$. Restoration of the CD 4 concentration has been demonstrated but the long-term best adaptive protocol is yet to be determined.

A mechanistic model of the evolution of CD4 after IL7 injections has been developed $^{1}$, which is based on a system of ordinary differential equations and includes random effects. Based on the estimation of this model, we use a Bayesian approach to forecast the dynamics of CD4 in new patients. We propose four prediction-based adaptive protocols of injections to minimize the time spent under $500 \mathrm{CD} 4$ cells $/ \mu L$ for each patient, without increasing the number of injections received too much. We show that our protocols significantly reduce the time spent under 500 CD4 over a period of two years, without increasing the number of injections. These protocols have the potential to increase the efficiency of this therapy.
\end{abstract}

\section{KEYWORDS:}

HIV, Interleukine 7, Adaptive protocols, Mechanistic models

\section{1 | INTRODUCTION}

Infection by the Human Immunodeficiency Virus (HIV) leads to a decrease of the concentration of CD4+ T lymphocytes (CD4) associated with a deficiency of the immune system, which increases the risk of opportunistic infections ${ }^{2}$. With an effective combination antiretroviral treatment (c-ART), the viral load becomes undetectable. The CD4 pool is then reconstituted in most cases $^{3}$. However, this does not happen for some patients ${ }^{4}$ who are low immunological responders. These patients, who present CD4 counts below 500 cells $/ \mu L$ of blood, have a lower life expectancy $[5$ and an increase of non-AIDS conditions, such as cancer or cardiovascular diseases ${ }^{6}$. To help the reconstitution of the CD4 pool, a treatment based on injections of exogenous Interleukin 7 (IL7) has been experimented. IL7 is a cytokine produced by thymus stromal cells and lymph nodes, and is involved in the CD4 
homeostasis ${ }^{718}$. Several trials have demonstrated the safety and the beneficial effect of exogenous IL7 on immune markers ${ }^{9110 / 11}$. Repeated cycles of three injections of IL 7 have been evaluated with the aim of maintaining the CD4 counts above 500 cells $/ \mu \mathrm{L}$ because, in this case, HIV infected subjects have about the same life expectancy as the general population ${ }^{12}$. In the phase I/II trials INSPIRE 2 and $3^{13}$, the repeated cycles of IL 7 could indeed maintain CD 4 concentration above the limit of 500 cells $/ \mu \mathrm{L}$ most of the time, although the best adaptive protocol is yet to be determined.

Mechanistic models, based on Ordinary Differential Equations (ODE), have been applied to model different infectious dis-

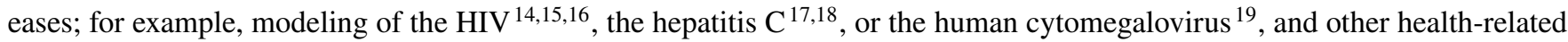
processes ${ }^{20}$. They have also been used to understand and predict the effects of IL7. A first work quantified the effect of exogenous IL7 on the proliferation rate of CD4 cells and showed an additional effect on the cells' survival ${ }^{21}$. The model was then extended to fit repeated injections ${ }^{1}$. Thanks to a population approach with random effects, the models were able to predict future individual responses to new injections of IL7 with a very good accuracy. This opened the opportunity to individualize the strategy of IL7 administration.

Dynamical adaptation of the treatment as a function of the response of the patient has been proposed by Murphy ${ }^{22}$ and Robins $\frac{23}{23}$ who developed the optimal treatment regime theory. Many papers have followed in this field 24 25/26/27]. Methods based

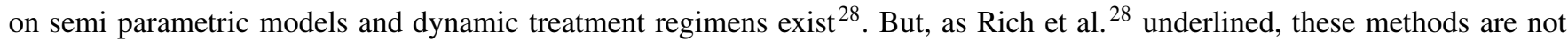
realistic enough and they often miss some important confounders. This issue can be solved by using mechanistic models ${ }^{29}$. When an ODE-based mechanistic model is available, the modeled treatment can be adapted using this model. This has been described by Rosenberg et al. ${ }^{30}$ for the supervised treatment interruption (STI) strategies, or in the pharmacokinetic-pharmacodynamic (PK/PD) field ${ }^{18 / 31}$. The optimal control theory can be applied for globally optimizing the treatment regime, which has been

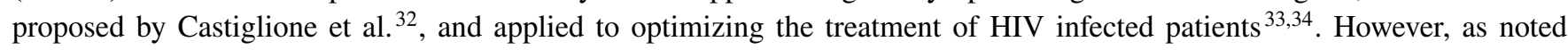
by Chakraborty and Murphy ${ }^{35}$ these works do not sufficiently take into account the statistical issues of the problem: model parameters have to be estimated and for efficient estimation, random effects have to be introduced in the statistical model. Such random effect mechanistic model has been applied to tune the dose of an antiretroviral treatment by Prague et al. .36 .

In the present paper, we aim to find efficient adaptive protocols for IL7 administration based on predictions from a random effect mechanistic model proposed in Jarne et al. 1 . Here, we propose realistic protocols that shorten the time spent under the limit of $500 \mathrm{CD} 4 \mathrm{cells} / \mu \mathrm{L}$ and limit the number of IL7 injections. Two approaches will be compared: adapting the criterion for a new cycle based on the risk of falling under $500 \mathrm{CD} 4 \mathrm{cells} / \mu \mathrm{L}$ before the next visit, and adapting the times of control visits. Both of these approaches are based on predictions generated with our random effect mechanistic model at relatively short-term to locally optimize the protocols. This is less ambitious than optimal control but is more feasible and the proposed protocols could soon be proposed to real patients. In both approaches we may or may not adapt also the number of injections per cycle, leading to four possible protocols.

The paper is organized as follows. Section 2 describes the data from INSPIRE 1, 2 and 3, and the design of the protocol for repeated cycles. Section 3 presents the mathematical and statistical features of the model. In Section 4, we describe two prediction-based adaptive protocols and their two variants adapting the number of injections per cycle or not. Section 5 presents the simulation study and its results while section 6 shows what the method would have predicted for real data. Section 7 concludes this paper.

\section{2 | DATA}

The data used for the mechanistic model are drawn from the INSPIRE 1, 2 and 3 studies 9113 . These studies evaluated the effect of injections of IL7 on the CD4 concentration in low immunological responders aged 18 years or more. The patients were included in the study if they were under stable c-ART for at least one year, had CD4 counts between 100 and 400 cells / $\mu L$ of blood, and had an undetectable viral load for at least 6 months before the beginning of the protocol.

The first study, INSPIRE 1 (initially called simply "INSPIRE"), evaluated the effect of one cycle of injection, which is defined as three injections with one week between each one. Three doses $(10,20$ and $30 \mu g / K g)$ were tested, and a placebo was included, for a total of 21 patients. The INSPIRE 2 and 3 studies evaluated the effect of repeated cycles of injections, using only the 20 $\mu \mathrm{g} / \mathrm{Kg}$, which was determined to be the most effective without too many side effects.

Overall, the data from 128 patients are used, with regular measurements of the CD4 counts and the marker of proliferation Ki67. The patients had visits at weeks 1, 2, 3, 4, 5, 6, 9 and 12, and then visited every 3 months. CD4 counts were measured at each visit, while Ki67 counts were measured only at weeks 1, 2, 3, 5 and 12. For the repeated cycles, the maintenance 

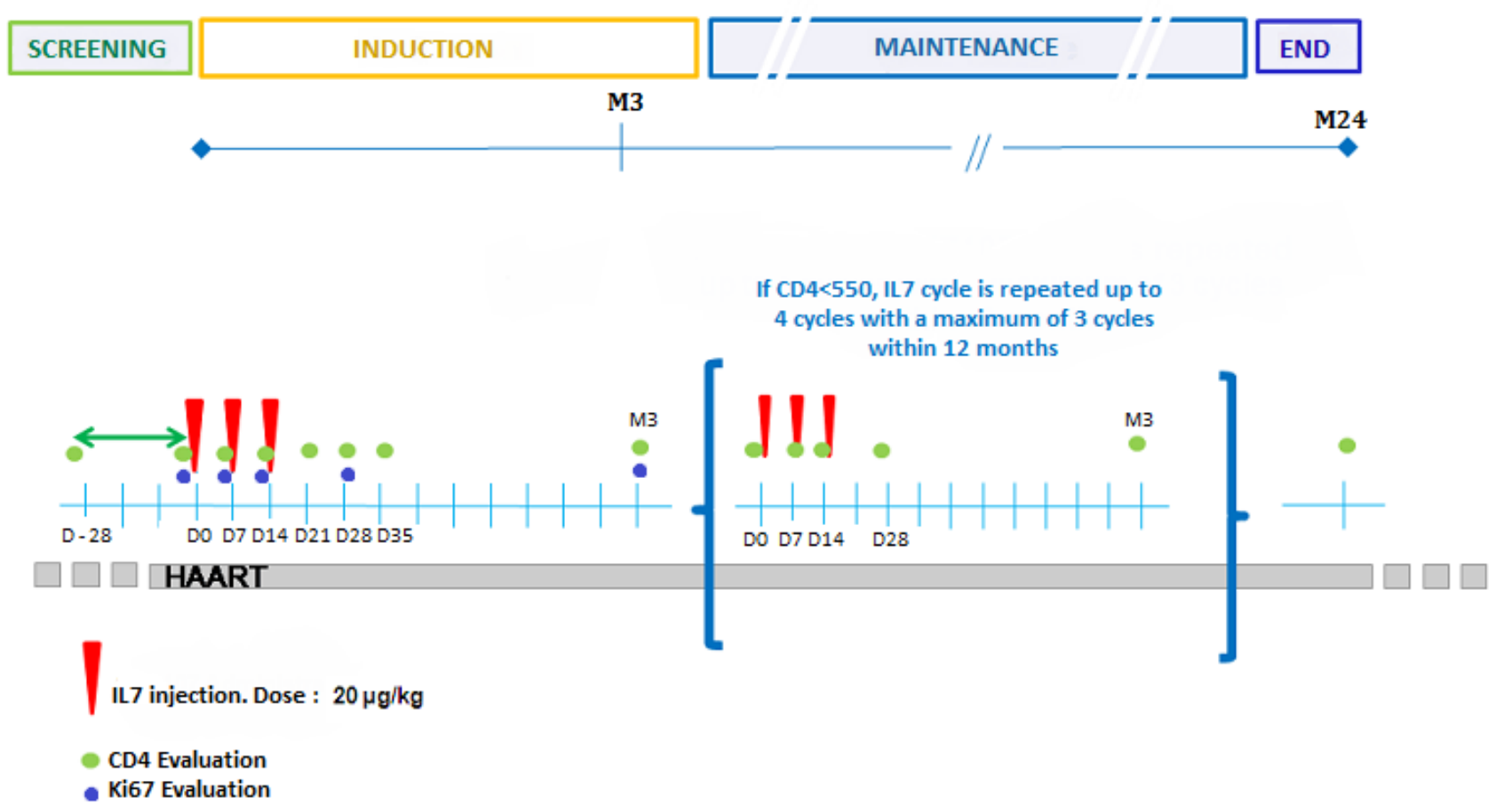

FIGURE 1 Design of INSPIRE 2 study. The screening phase determines the concentrations of CD4 at baseline to include or not the patient. The induction phase starts with a first cycle of three IL7 injections at weeks 1,2 and 3. The maintenance phase then controls the patient every 3 months with a new cycle if the patient is under $550 \mathrm{CD} 4$. The green dots represent the measures of CD4 counts, blue dots the measures of Ki67.

phase consisted in repeated visits every three months. When the CD4 counts were below 550, a new cycle of injections was administered. The durations of the studies were 12, 24 and 21 months for INSPIRE, INSPIRE 2 and 3, respectively. The design of INSPIRE 2 is presented in Figure 1

The high heterogeneity of the data in terms of number of injections per cycles, number of cycles received and dose, and the availability of regular measurements of different immunological markers makes this an interesting dataset for modeling. Overall, 197 cycles were administered for 128 patients, with 41 incomplete cycles (one or two injections instead of three). Because the patients had different trajectories of CD4 counts, they had different times of injections in the maintenance phase.

\section{3 | MECHANISTIC MODEL FOR IL7 TREATMENT}

\section{1 | Modeling of the effect of IL7 injections on CD4 concentration}

A mechanistic model for the evolution of the CD4 concentration after repeated IL7 injections was proposed in Jarne et al. ${ }^{1}$. In this paper, a two compartment model was described, with the compartment $P$ for the proliferating cells and the compartment $Q$ for the quiescent cells. This model and the different effects on the parameters have been selected with the LCVa criterion ${ }^{377}$, as described in Thiebaut et al. ${ }^{21}$ and Jarne et al. ${ }^{[1}$. The mathematical structure of the model is written as:

$$
\left\{\begin{array}{l}
\frac{d Q}{d t}=\lambda+2 \rho P-\pi Q-\mu_{Q} Q \\
\frac{d P}{d t}=\pi Q-\rho P-\mu_{P} P
\end{array}\right.
$$

We allow the parameters to be different from one subject to another. Thus, we denote by $\xi^{i}$ the vector of parameters of the ODE system: $\xi^{i}=\left[\lambda^{i}, \rho^{i}, \pi^{i}, \mu_{Q}^{i}, \mu_{P}^{i}\right]$ for patient $i(i=1, \ldots, n)$ and by $X^{i}=\left(Q\left(t, \xi^{i}\right), P\left(t, \xi^{i}\right)\right)$ his state vector. All of the parameters are positive because they are rates of proliferation, production and death for cells. Hence, we use a log transformation denoted by a tilde: $\tilde{\xi}_{l}^{i}=\log \left(\xi_{l}^{i}\right)$. The meaning and units of each parameter are detailed in Table 1 
The initial condition for subject $i$ is the equilibrium point: $\frac{d Q}{d t}\left(0, \xi^{i}\right)=0, \frac{d P}{d t}\left(0, \xi^{i}\right)=0$, which gives the initial points: $Q\left(0, \boldsymbol{\xi}^{i}\right)=\frac{\lambda^{i}\left(\rho^{i}+\mu_{P}^{i}\right)}{\pi^{i}\left(\mu_{P}^{i}-\rho^{i}\right)+\mu_{O}^{i}\left(\rho^{i}+\mu_{P}^{i}\right)}, P\left(0, \boldsymbol{\xi}^{i}\right)=\frac{\lambda^{i} \pi^{i}}{\pi^{i}\left(\mu_{P}^{i}-\rho^{i}\right)+\mu_{O}^{i}\left(\rho^{i}+\mu_{P}^{i}\right)}$.

A patient-by-patient inference is inefficient if there is not enough information for each subject; hence, we need a statistical model of the variability of the parameters using both explanatory variables with fixed effects and random effects. It can be written as:

$$
\left\{\begin{aligned}
\tilde{\lambda}^{i}(t) & =\tilde{\lambda}_{0}+l_{i} \\
\tilde{\pi}^{i}(\mathrm{t}) & =\tilde{\pi}_{0}+\left[\beta_{C^{\prime}} \mathbb{1}_{\left\{C^{i}(t)>1\right\}}+\sum_{k=1}^{3} \mathbb{1}_{\left\{N_{t}^{i}=k\right\}} \beta_{\pi_{k}} d_{i}^{0.25}\right] \mathbb{1}_{\left\{N_{t}^{i}-N_{t-7}^{i}=1\right\}} \\
\tilde{\rho^{i}(t)} & =\tilde{\rho}_{0}+r_{i} \\
\tilde{\mu_{Q}^{i}}(t) & =\tilde{\mu}_{Q_{0}}+\beta_{\mu_{Q}} f^{i}(t) d_{i}^{0.25} \\
\tilde{\mu_{P}^{i}}(t) & =\tilde{\mu}_{P_{0}}
\end{aligned}\right.
$$

where $l_{i}$ and $r_{i}$ are normally distributed random effects: $l_{i} \sim \mathscr{N}\left(0, \sigma_{\lambda}^{2}\right)$ and $r_{i} \sim \mathscr{N}\left(0, \sigma_{\rho}^{2}\right) ; C^{i}(t)$ counts the number of cycles and $\beta_{C}$ represents the cycle effect, meaning that after one cycle the effect of IL7 on the proliferation can be lower; $\beta_{\pi_{k}}$ is the effect of the injection on the proliferation, $k$ being 1,2 or 3 depending on whether the injection is the first, second or third of the cycle. The effects on $\pi$ are constant during 7 days after each injection, and they then disappear. $N_{t}^{i}$ counts the number of injections that patient $\mathrm{i}$ has received until time $\mathrm{t}$; thus, $\mathbb{1}_{\left\{N_{t}^{i}-N_{t-7}^{i}=1\right\}}$ is an indicator function taking the value 1 if an injection was administered in the last 7 days. Let $T_{t}^{i}$ be the time of the last injection received by the patient $i$ at the time $t$, the effect on $\mu_{Q}$ is represented by $\beta_{\mu_{Q}} f(t)$, with $f$ written as:

$$
f^{i}(t)= \begin{cases}1 & \text { if } 2+T_{t}^{i}<t \leq 360+T_{t}^{i} \\ 1-(t-360) / 360 & \text { if } 360+T_{t}^{i}<t \leq 720+T_{t}^{i} \\ 0 & \text { if } 720+T_{t}^{i}<t\end{cases}
$$

In our data, the variable $P$ and $Q$ are not directly observed. The observations correspond to the total number of CD4 and the number of proliferating cells; hence, we also need an observation model. The observation of $M$ biomarkers are related to the solution of the ODE with the function $\mathrm{g}: Y_{m q}^{i}=g_{m}\left(\xi^{i}, t_{q}\right)^{0.25}+\epsilon_{m j}^{i}, m=1, \ldots, M$. Here $M=2$ as we observe the CD4 counts and the Ki67 counts. Denoting by $Y_{1 j}^{i}$ the fourth root of the CD4 counts, and by $Y_{2 k}^{i}$ the Ki67 counts for patient $i$ at times $t_{i j}$ $\left(j=1, \ldots, J^{i}\right)$ and $t_{i k}\left(k=1, \ldots, K^{i}\right)$, respectively. This gives the following observation model:

$$
\begin{cases}\mathrm{Y}_{1 j}^{i}=\left[P\left(t_{i j}, \xi^{i}\right)+Q\left(t_{i j}, \xi^{i}\right)\right]^{0.25} & +\epsilon_{1 j}^{i} \\ \mathrm{Y}_{2 k}^{i} & =P\left(t_{i k}, \xi^{i}\right)^{0.25}\end{cases}
$$

We assume that noises variables are normally distributed: $\epsilon_{1 j}^{i} \sim \mathscr{N}\left(0, \sigma_{1}^{2}\right), \epsilon_{2 k}^{i} \sim \mathscr{N}\left(0, \sigma_{2}^{2}\right)$. This parameter includes the measurement error and the biological variation not taken into account in the model; for example, the diurnal variation of the CD4 concentration. The fourth-root transformation has been applied to make homoscedasticity and normality acceptable 21 .

\section{2 | Inference and estimation}

To estimate the parameters of the model, Jarne et al.$^{1}$ used the NIMROD program ${ }^{38}$. The implemented estimation method is based on penalized log-likelihood maximization. This is a quasi-Bayesian approach in the sense that penalization is built from a priori values for the parameters found in the literature. Then, this penalized log-likelihood is maximized using a Newton-Raphson like algorithm, called Robust Variance Scoring ${ }^{39}$. The iterative algorithm stops when the Relative Distance to Maximum (RDM) criterion is satisfied. The results of this inference are displayed in Table 1 There are enough observations to consider that the posterior distributions are close to normal distribution (in virtue of the Bernstein-von Mises Theorem ${ }^{40}$ ), so that results in Table 1 can be interpreted as summarizing the (marginal) posterior distributions of the parameters by their expectations and their standard deviations. 
TABLE 1 Parameters of the model and their estimates from Jarne et al. 1

\begin{tabular}{llll}
\hline \hline Parameter & Name & Units & $\begin{array}{l}\text { Estimate } \\
\text { mean (sd) }\end{array}$ \\
\hline$\lambda$ & Production rate & cells.days $^{-1}$ & $5.32(0.33)$ \\
$\rho$ & Reversion rate & days $^{-1}$ & $2.44(0.23)$ \\
$\pi$ & Proliferation rate & days $^{-1}$ & $0.06(0.004)$ \\
$\mu_{P}$ & Death rate of P cells & days $^{-1}$ & $0.07(0.005)$ \\
$\mu_{Q}$ & Death rate of Q cells & days $^{-1}$ & $0.08(0.02)$ \\
\hline$\beta_{\pi_{1}}$ & Effect of IL7 on $\pi$ (Injection 1) & days $^{-1} \cdot \mu g^{-1}$ & $0.93(0.04)$ \\
$\beta_{\pi_{2}}$ & Effect of IL7 on $\pi$ (Injection 2) & days $^{-1} \cdot \mu g^{-1}$ & $0.71(0.04)$ \\
$\beta_{\pi_{3}}$ & Effect of IL7 on $\pi$ (Injection 3) & days $^{-1} \cdot \mu g^{-1}$ & $0.23(0.04)$ \\
$\beta_{\mu_{Q}}$ & Effect of IL7 on $\mu_{Q}$ & days $^{-1} \cdot \mu g^{-1}$ & $-0.08(0.006)$ \\
$\beta_{C}$ & Cycle effect of IL7 & days $^{-1}$ & $-0.16(0.02)$ \\
\hline$\sigma_{\lambda}$ & Standard deviation of random effect on $\lambda$ & cells.days $^{-1}$ & $0.24(0.03)$ \\
$\sigma_{\rho}$ & Standard deviation of random effect on $\rho$ & days $^{-1}$ & $0.52(0.08)$ \\
\hline$\sigma_{1}$ & Noise parameter on CD4 cells & cells $^{0.25}$ & $0.29(0.003)$ \\
$\sigma_{2}$ & Noise parameter on P cells & cells $^{0.25}$ & $0.28(0.02)$ \\
\hline
\end{tabular}

\section{4 | PREDICTION-BASED ADAPTIVE PROTOCOLS}

\section{1 | General method}

In the original protocol of INSPIRE 2 and 3 studies, which is called the "ORI" protocol, patients start a first cycle of injections, with CD4 counts measurements at the times of injections. There are visits every 3 months. A new cycle is administered if the CD4 counts are below $550 \mathrm{CD} 4$. The aim is to prevent CD4 concentration to fall under 500. Thus, the original protocol is already an adaptive protocol but because the patients have different CD4 dynamics, this fixed criterion is not always appropriate. If a patient tends to return quickly to his or her baseline concentration, then the margin can be too small and the decision not to administer a new cycle can lead to cross the limit of $500 \mathrm{CD} 4$ shortly after the control. In contrast, for some patients the CD4 concentration decreases slowly after an injection, and the criterion of 550 CD4 for a new cycle may be too high, which results in unnecessary cycles and visits. Here, we propose protocols that are based on the prediction that can be done with a mechanistic model with the aim of decreasing the time spent under the limit of $500 \mathrm{CD} 4$, while controlling the number of IL7 injections.

We use a mechanistic model that fits the dynamics of CD4 following a cycle of IL7 injections; the fixed effects parameters have been estimated using all observations of the three INSPIRE studies. For the mixed effect parameters, $\lambda$ and $\rho$, we have an estimation of the mean of the parameters on the population, and the variance of their random effects $\left(\sigma_{\lambda}^{2}\right.$ and $\left.\sigma_{\rho}^{2}\right)$. With these estimates and the informations for the patient $i$ available at the time $k$, called $H_{i}^{t_{k}}$, we used an MCMC algorithm to sample the posterior distribution of the individual parameters $\lambda^{i}$ and $\rho^{i}$. For given values of the parameters of a patient, we can predict the evolution of his or her CD4 concentration ${ }^{36}$. Taking into account the uncertainty on the parameters, we can also compute the distribution of any quantity related to the future $\mathrm{CD} 4$ concentration dynamic. This prediction can be used to adapt the treatment. Every time that the patient comes for a control visit, we have access to new data so that $H_{i}^{t_{k}} \subset H_{i}^{t_{k+1}}$; thus, the prediction is more precise as time goes on.

The algorithm used for sampling the random effects of the patient is a Metropolis Within Gibbs ${ }^{41}$. At each iteration, $\lambda$ and $\rho$ are successively sampled. In the Metropolis part of the algorithm, the instrumental function used for the first estimation of the protocol ( 3 month control) is the posterior law estimated with NIMROD. The standard error of the prior for $\lambda$ and $\rho$ is the one estimated with NIMROD (respectively, $s d_{\lambda}=0.33$ and $s d_{\rho}=0.23$, see Table 1 added with the standard error associated with the random effect (respectively, $\sigma_{\lambda}=0.24$ and $\sigma_{\rho}=0.52$, see Table 1 , as the variability comes from both the error of estimation and the inter-individuals variability. Then, for each control visit after the first one, the distribution given by the previous MCMC is used as the new prior. The likelihood used in the MCMC procedure is ${ }^{42}$. 


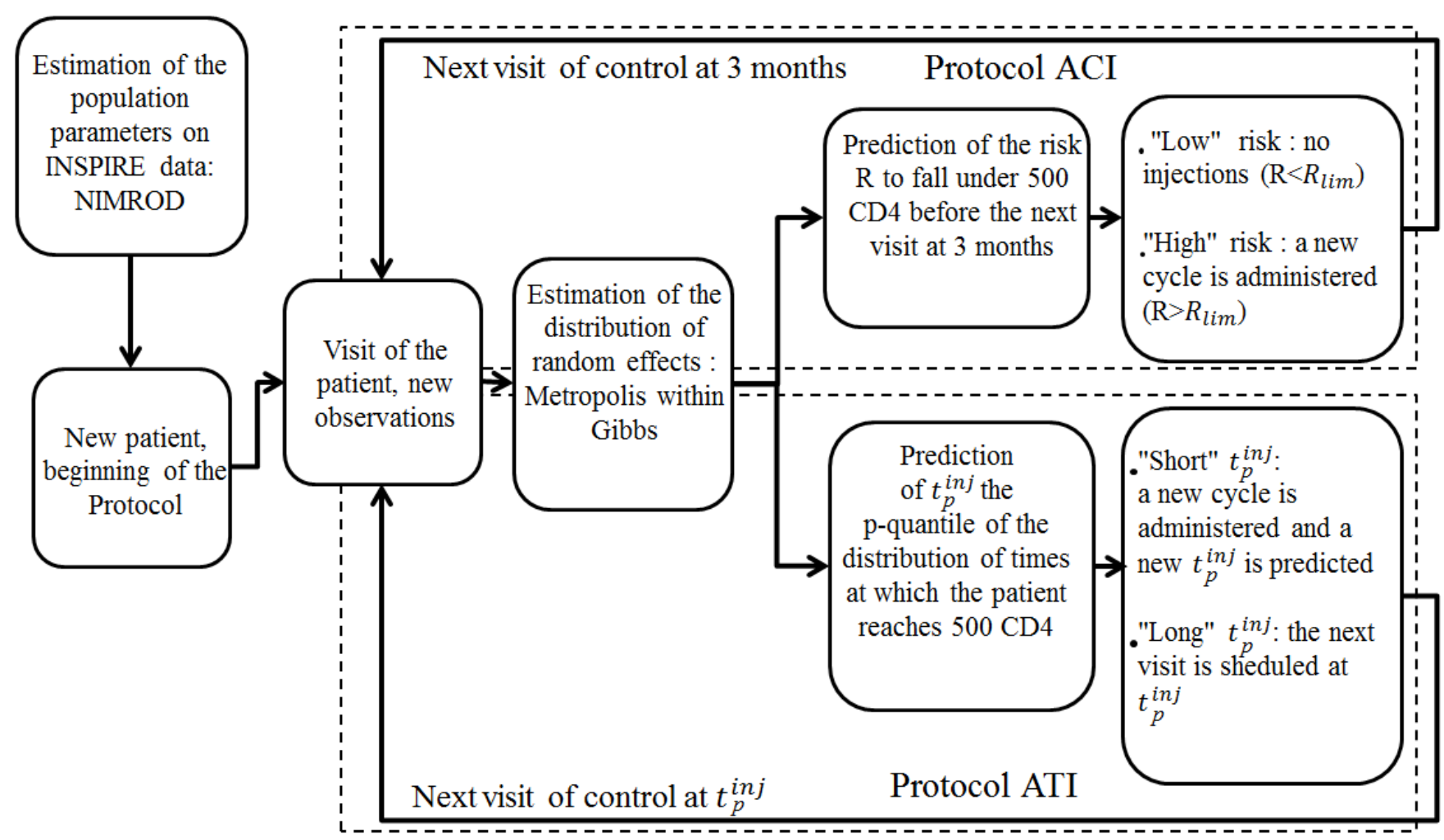

FIGURE 2 Flowchart of the two protocols. ACI Protocol: Adaptive Criterion Protocol, the visits are every 3 months and the decision to administer a new cycle is based on the predicted risk $R$ to fall under 500 CD4 before the next visit. ATI Protocol: Adaptive Times Protocol, the times of visit are predicted based on the time at which the patient is supposed to reach the 500 CD4 limit, and a new cycle is administered if this predicted time is too short.

$$
L_{i}=\prod_{m=1}^{M} \prod_{j=1}^{K_{m}} \frac{1}{\sigma_{m} \sqrt{2 \pi}} \exp \left[-\frac{1}{2}\left(\frac{\mathrm{Y}_{\mathrm{mj}}^{\mathrm{i}}-\mathrm{g}_{\mathrm{m}}\left(\mathrm{t}_{\mathrm{j}}, \xi^{\mathrm{i}}\right)^{0.25}}{\sigma_{\mathrm{m}}}\right)^{2}\right]
$$

As presented in van der Vaart ${ }^{40}$, according to Doob's theorem, the distribution of the parameters $\hat{\xi}^{i}$ with the data from patient $i$ at time $t_{k}\left(H_{i}^{t_{k}}\right)$ converges to the Dirac of the true value of the parameters, $\delta\left(\xi^{i}\right)$, when $k$ tends to infinity.

We propose two prediction-based adaptive approaches; the first is based on an adaptive criterion of injections (ACI); the second is based on an adaptive time of injections (ATI). Both approaches have a variant where the number of injections per cycle can be adapted. Figure 2 presents the flowchart of the two approaches.

\section{2 | Prediction ability on real data}

Before developing and studying prediction-based adaptive protocols, it is essential to study the prediction ability of our model. In this aim, we randomly excluded 10 patients from the data and estimated the parameters of the model on the reduced dataset. For each excluded patient, we ran the MCMC algorithm using the estimates from the diminished dataset. For an excluded patient $i$, at each time of control (every three months), the MCMC algorithm gives a distribution of CD4 concentration at every time of observation $t_{j}$. At each iteration q of the MCMC, we can generate the predicted distribution of observation at each time $t_{i j}$ by computing $\mathrm{CD} 4_{j q}^{i}=P\left(t_{i j}, \xi^{i q}\right)+Q\left(t_{i j}, \xi^{i q}\right)$, and adding a noise variable: $\mathrm{Y}_{1 j q}^{i}=\left(\mathrm{CD} 4_{j q}^{i}\right)^{0.25}+\epsilon_{1 j q}^{i}$. Figure 3 shows examples of these predicted distributions for four (out of the 10) patients. The black line represents the mean of the future trajectories and the light blue band represents $95 \%$ credible intervals; the dark blue band represents $95 \%$ predictive interval of observations, and the black dots are the observed values. The $95 \%$ credible and predictive intervals were computed by excluding the $2.5 \%$ most extreme values of each side of the distribution. If the model is well calibrated, then the black dots should be inside the dark blue band, which is the case here. 

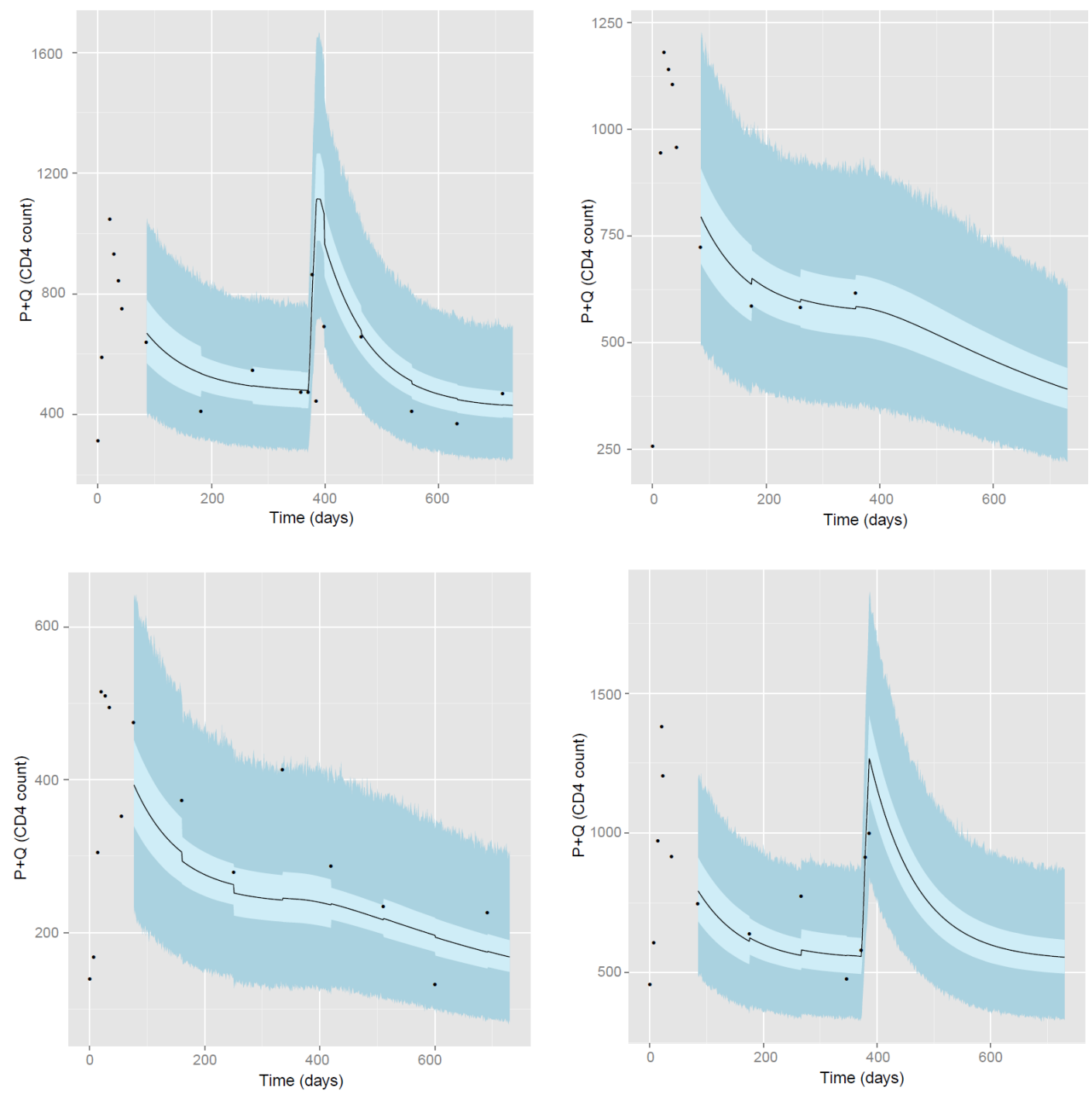

FIGURE 3 Prediction intervals for four random patients. Light blue band : 95\% credible interval of trajectories. Dark blue band : 95\% predictive interval of observations. Black line : mean of predicted trajectories. Black dots: real data observations.

To show the good calibration of the predicted distributions, a quantile analysis was done. The process of studying the prediction for 10 excluded patients was repeated 10 times leading to a total of 100 patients, and we analyzed the distributions of the observed data with respect to the predicted distributions by a QQ-plot. The quantiles of the predicted distributions should be the same as the observed quantiles, which was indeed essentially the case, as shown in the QQ-plot presented in the web supplementary 1.

\section{3 | Protocol with Adaptive Criterion of Injections (ACI)}

The ACI protocol is similar to the original protocol: the patients come every three months for a control visit. However, instead of using the fixed criterion of CD4 counts below 550, we predict the risk $R$ that the CD4 concentration will fall below 500 before the next visit. With the distribution of parameters given by the MCMC algorithm presented in Section 4.1 we can directly have the distribution of CD4 concentration at three months, which allows us to compute $R$ :

$$
R\left(\hat{\xi}^{i} \mid H_{i}^{t_{k}}\right)=\mathrm{P}\left(\mathrm{g}_{1}\left(\mathrm{t}_{\mathrm{k}}+\mathrm{t}_{\mathrm{visit}}, \hat{\xi}^{\mathrm{i}}\right)>500 \mid \mathrm{H}_{\mathrm{i}}^{\mathrm{t}_{\mathrm{k}}}\right),
$$


where $t_{\text {visit }}$ is the time between two controls (three months in INSPIRE studies). If $R$ is larger than a limit risk called $R_{\lim }$ (for instance, 10\%), a new cycle of injections is administered. If $R<R_{\lim }$ then the patients simply comes back three months later. At each visit, the MCMC algorithm is done with new data, and the decision is made with the value of the risk $R$.

\section{4 | Protocol with Adaptive Times of Injections (ATI)}

In the ATI protocol, the injections times are adaptive. The patient starts his or her first cycle and then comes back 3 months later. We denote $t^{\text {inj }}$ such as $g_{1}\left(t^{\mathrm{inj}}, \widehat{\boldsymbol{\xi}}^{i}\right)=500$, the time at which the patient will reach the $500 \mathrm{CD} 4$ limit. Then, with the MCMC algorithm, we sample the distribution of $t^{\mathrm{inj}}$ and select $t^{\mathrm{inj}_{\mathrm{p}}}$ as the $p$ quantile (for instance the 0.1 quantile) of this distribution: $\mathscr{P}\left(t^{\mathrm{inj}}<t_{p}^{\mathrm{inj}}\right)=p$. If $t_{p}^{\mathrm{inj}}$ is larger than a fixed limit (here 1 month), the patient comes for a new visit at the time $t_{p}^{\mathrm{inj}}$. If not, then a new cycle is administered immediately and the next time of visit is computed again at the end of the cycle. The time between two cycles is chosen here to be at least one month because it is clinically relevant.

\section{5 | Adaptive number of injections: ACIC and ATIC protocols}

Up to now, the proposed protocols used cycles of three injections. In Jarne et al.1 questioned: the estimation of the effect of this injection, $\beta_{\pi 3}$, showed that the impact of this injection on the proliferation rate is much smaller compared to the first two injections. A comparison of the simulation of protocols showed that the original protocol with only two injections per cycle reduced the number of injections without impacting the time spent under 500 CD4 or the mean number of CD4. We propose a modification of the ACI and ATI protocols for adapting the number of injections per cycle, $\mathrm{a} \mathrm{C}$ is added in the end of their names to represent the adaptation inside a cycle, leading to the ACIC and ATIC protocols:

- Protocol ACIC: when a decision for a new cycle is taken, the aim is to decide if the decision to diminish the number of injections will change the decision to inject or not at the next time of control. To do this, we compare the risks to fall under $500 \mathrm{CD} 4$ at the next control visit for a cycle of three, two or one injection: $R\left(\hat{\xi}_{j}^{i} \mid H_{i}^{t_{k}}\right)=\mathscr{P}\left(g_{1}\left(\hat{\xi}_{j}^{i}, t_{k}+2 t_{\mathrm{visit}}\right)>500 \mid H_{i}^{t_{k}}\right)$, with $j(j=1, \ldots, 3)$ being the number of injections of the cycle, and $\hat{\boldsymbol{\xi}}_{j}^{i}$ depending on this number of injection as presented in Equation 11 . If in any case a cycle of injections would be necessary at the next time of visit, meaning that $R\left(\hat{\xi}_{3}^{i} \mid H_{i}^{t_{k}}\right)$ is superior to the chosen $R_{\lim }$, then we consider that the patient needs a cycle of three injections. If this is not the case, and if both $R\left(\widehat{\xi}_{2}^{i} \mid H_{i}^{t_{k}}\right)$ and $R\left(\hat{\xi}_{1}^{i} \mid H_{i}^{t_{k}}\right)$ are inferior to $R_{\mathrm{lim}}$, then a cycle of one injection is administered. If $R\left(\hat{\xi}_{2}^{i} \mid H_{i}^{t_{k}}\right)$ is inferior to $R_{\lim }$ but not $R\left(\hat{\xi}_{1}^{i} \mid H_{i}^{t_{k}}\right)$, a cycle of two injections is administered. And if only $R\left(\hat{\xi}_{3}^{i} \mid H_{i}^{t_{k}}\right)$ is inferior to $R_{\lim }$, a cycle of three injections is administered.

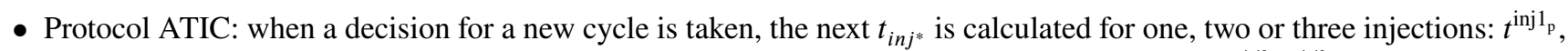
$t^{\mathrm{inj} 2_{\mathrm{p}}}$ and $t^{\text {inj }^{\mathrm{n}} \mathrm{p}_{\mathrm{p}}}$. The relative difference between two and three injections is calculated: $d_{3}=\frac{t^{\mathrm{inj} \mathrm{j}_{\mathrm{p}}} t^{\mathrm{inj} 3_{\mathrm{p}}}}{t^{\mathrm{inj} 3_{\mathrm{p}}}}$. If $d_{3}$ is superior to $d_{\text {lim }}$, then three injections are administered. If not, then the same process is repeated to choose between one or two

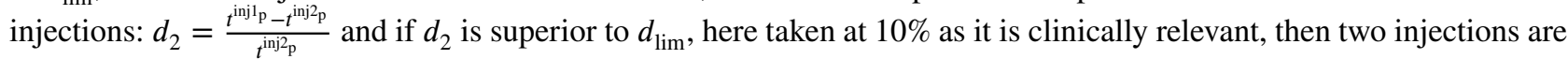
administered; if not, then one injection is administered.

\section{5 | SIMULATION}

\section{1 | General description}

We simulated the different protocols for 150 "pseudo-patients" on a two-year period. The parameter values of these patients were sampled from the posterior distribution of the parameters estimated with NIMROD over the 138 patients of the INSPIRE studies. Because $\lambda$ and $\rho$ vary between patients, random effects were generated for all patients for these parameters, using the estimated variance of the random effects on the population. Moreover, we applied the inclusion criterion of the INSPIRE studies, keeping only those patients with baseline CD4 counts between 100 and 400. The simulations of the trajectories were done with $\mathrm{R}$ with the DeSolve package ${ }^{43}$. This package numerically solves the ODE for a given set of parameter values $\xi^{i}$, using the "lsodes" method, an interface to the FORTRAN ODE solver bearing the same name ${ }^{44}$. Observations were generated at times $(0$, $7,14,21,55)$ before the first time of control (day 90) by adding a noise variable (according to Equation (2)) to the value of the trajectories at these times. Each time that we make a decision, the observations are generated to take into account the decision: 
if a cycle is administered, then the next observations are at the time of injections and at the next time of control; if not, then the next generated observation is only at the next time of control. The total number of CD4 and the number of proliferating cells are observed each time.

For the ACI and ACIC Protocols, the values of the risk limit $R_{\mathrm{lim}}$ used were $0.05,0.1$ and 0.2. For the ATI and ATIC Protocols, we used the $0.05,0.1$ and 0.2 quantiles to obtain $t_{p}^{\mathrm{inj}}$. The MCMC algorithm was performed with a total of 5000 iterations each time, with a burn-in phase of 1000 and a thinning of 2 . The convergence of the chain was controlled with the Heidel diagnostic, the results of the convergence are presented in web supplementary 2 .

\section{2 | Results for the adaptive protocols}

To compare the different protocols, we used eight criteria: the mean number of CD4 over the protocol, the time spent under 500 CD4, the number of cycles administered, the number of visits (including the visits for the injections inside a cycle), the number of injections, the number of cycles of one, two and three injections (the number of cycles of three injections includes the first cycle, which is not decided by the protocol because it is the start of the protocol and automatically of three injections). The results are presented in Table 2 We considered the value of $R_{\text {lim }}$ (for ACI and ACIC) and the $p$ quantile (for ATI and ATIC) as "threshold" because they both represent the limit value for the decision.

Compared to the original protocol (ORI), the proposed protocols highly reduce the time spent under 500 CD4, while the number of visits is comparable (slightly increased for the ACI protocol, and decreased for the ATI and ATIC protocols). The number of injections is increased for the ACI protocol but this increase is not as important when the number of injections per cycle is also adapted (ACIC) while the time spent under 500 CD4 is similar in both ACI and ACIC protocols. The number of injections is similar between the ATI and ORI protocols, while it is reduced by the ATIC protocol.

The mean of CD4 is higher for the ACI and ACIC protocols than the ATI and ATIC protocols (which are similar to the ORI protocol), because the aim of the ATI and ATIC protocols is to start a cycle just before the patient reaches 500 CD4, while the ACI and ACIC protocols have visits of control only every 3 months, which means that the decision to start a new cycle can be taken while the patient could wait before he or she reaches 500 CD4 but not 3 months. This means that when a new cycle is started, we expect CD4 concentration to be around 500 in the ATI and ATIC protocols, while these concentrations can be higher in the ACI and ACIC protocols. This induces a higher mean of CD4 for ACI and ACIC protocols. This is consistent with the choice of the criterion of interest, namely the time spent with CD4 concentrations under 500. This choice is justified by clinical results $\frac{12}{}$ and consistency with INSPIRE studies. However, our method can be modified by using other criteria. If it is clinically relevant to consider the mean number of CD4, it could be easily implemented in the decision criterion for adapting the protocol.

The sensitivity analysis on the threshold parameter shows that for the ACI and ACIC protocols, the risk $R_{\text {lim }}$ does not significantly impact the results in any of the criteria. For the ATI and ATIC protocols, the $p$-quantile at 0.05 does not increase the frequency of cycles or visits but it does reduces the time spent under 500 CD 4 on average. The $p$-quantile 0.2 is no better because it increases the time spent under 500 CD4 but does not reduce the number of cycles.

Figure 5 presents the boxplot of the time spent under $500 \mathrm{CD} 4$, the number of visits, the mean of CD4 and the number of injections for each protocol at the threshold 0.05. This shows that the median of time spent under $500 \mathrm{CD} 4$ is at 0 for each of the proposed protocols. The ATI and ATIC protocols have higher 3rd-quantiles than the ACI and ACIC protocols but the outliers are smaller. This means that the ATI and ATIC protocols are more suitable for patients with difficulties to maintain their CD4 concentrations above 500. Indeed, the ATI and ATIC protocols allow more frequent visits for patients with a fast decrease of CD4 after the end of a cycle compared to the ACI and ACIC protocols, where a minimum delay of 3 months between visits has to be respected. Globally, it is clear that all four protocols have better results for the time spent under $500 \mathrm{CD} 4$ than the ORI Protocol. We can also see that all protocols have similar distributions for the three other criteria, but the ACI and ACIC induce higher CD4 means, and the ATIC is lower in number of visits and number of injections.

Figure 4 shows the plot of the dynamic of CD4 for three protocols (ORI, ACI and ATI) with the threshold 0.1 for two pseudopatients. In this figure, the real trajectory is represented by a dark line and the simulated observations are the black dots. The ORI protocol uses those observations for the decision to administer a new cycle with the criterion of 550 CD4. The proposed adaptive protocols predict a distribution of the random effects, which gives a distribution of CD4 at each time point using those observations. The adaptive protocols use those predicted distributions for the decisions while the ORI protocol relies only on the observation at the current time of control. The $95 \%$ credible interval of CD4 is represented by the light blue band, and the decision taken by the proposed adaptive protocols are based on this prediction. For each of those distributions of CD4, the distribution of the observations is also predicted by adding a noise variable to the predicted CD4, as it was done in Section 4.2 The $95 \%$ 
TABLE 2 Comparison of the Protocols ORI (original), ACI, ACIC, ATI and ATIC. Eight criteria are presented : the mean number of CD4 (CD4), the time spent under $500 \mathrm{CD} 4$ (T500), the number of cycle (Nb Cycles), the number of visits (Nb visits), the number of injections ( $\mathrm{Nb}$ inj), the number of cycle of respectively one, two and three injections ( $\mathrm{C} 1, \mathrm{C} 2$ and $\mathrm{C} 3)$. These are calculated per patient over the two year protocols, and their means and quartiles (or standard error for the mean of CD4) are presented."Threshold" means $R_{\lim }$ (for ACI and ACIC) or $p$ quantile (for ATI and ATIC).

\begin{tabular}{|c|c|c|c|c|c|c|c|c|c|}
\hline Protocol & Threshold & $\begin{array}{l}\text { CD4 } \\
\text { mean (sd) }\end{array}$ & $\begin{array}{c}\text { T500 (days) } \\
\text { mean }[01 ; 03]\end{array}$ & $\begin{array}{c}\mathrm{Nb} \text { Cycles } \\
\text { mean }[01 ;: Q 3]\end{array}$ & $\begin{array}{c}\mathrm{Nb} \text { visits } \\
\text { mean }[01 ; 03]\end{array}$ & $\begin{array}{l}\mathrm{Nb} \text { inj } \\
\text { mean [[1;:Q3] }\end{array}$ & $\begin{array}{c}C_{1} \\
\text { mean }[Q 1 ; 03]\end{array}$ & $\begin{array}{c}C_{2} \\
\operatorname{mean}[Q 1 ; 03]\end{array}$ & $\begin{array}{c}C_{3} \\
\operatorname{mean}[[1 ; 03]\end{array}$ \\
\hline ORI & - & $722(112)$ & $107[6 ; 173]$ & $4.5[3 ; 6]$ & $18[15 ; 20]$ & $13[9 ; 18]$ & 0 & 0 & $4.5[3 ; 6]$ \\
\hline \multirow{3}{*}{ ACI } & 0.05 & $882(146)$ & $18[0 ; 7]$ & $5.9[4 ; 8]$ & $20[17 ; 24]$ & $18[12 ; 24]$ & 0 & 0 & $5.9[4 ; 8]$ \\
\hline & 0.1 & 866 (143) & $19[0 ; 18]$ & $5.8[4 ; 8]$ & $20[17 ; 24]$ & $17[12 ; 24]$ & 0 & 0 & $5.8[4 ; 8]$ \\
\hline & 0.2 & $837(137)$ & $21[0 ; 25]$ & $5.6[4 ; 8]$ & $20[17 ; 24]$ & $17[12 ; 24]$ & 0 & 0 & $5.6[4 ; 8]$ \\
\hline \multirow{3}{*}{ ACIC } & 0.05 & 853 (124) & $17[0 ; 7]$ & $6.1[5 ; 8]$ & $18[12 ; 24]$ & $15[8 ; 24]$ & $0.8[0 ; 2]$ & $1.3[0 ; 2]$ & $4.0[1 ; 8]$ \\
\hline & 0.1 & $830(146)$ & $19[0 ; 20]$ & $6.0[5 ; 8]$ & $18[12 ; 24]$ & $15[7 ; 24]$ & $0.9[0 ; 2]$ & $1.2[0 ; 2]$ & $3.9[1 ; 8]$ \\
\hline & 0.2 & 804 (130) & $23[0 ; 26]$ & $5.8[4 ; 8]$ & $17[11 ; 23]$ & $14[7 ; 23]$ & $0.9[0 ; 2]$ & $1.1[0 ; 2]$ & $3.8[1 ; 7]$ \\
\hline \multirow{3}{*}{ ATI } & 0.05 & $785(71)$ & $13[0 ; 18]$ & $5.0[4 ; 7]$ & $16[13 ; 20]$ & $15[12 ; 21]$ & 0 & 0 & $5[4 ; 7]$ \\
\hline & 0.1 & $764(67)$ & $19[0 ; 32]$ & $4.9[3 ; 6]$ & $15[12 ; 18]$ & $15[9 ; 18]$ & 0 & 0 & $4.9[3 ; 6]$ \\
\hline & 0.2 & $743(64)$ & $28[0 ; 48]$ & $4.7[3 ; 6]$ & $15[11 ; 18]$ & $14[9 ; 18]$ & 0 & 0 & $4.7[3 ; 6]$ \\
\hline \multirow{3}{*}{ ATIC } & 0.05 & $769(72)$ & $16[0 ; 24]$ & $5.4[4 ; 7]$ & $12[10 ; 14]$ & $12[8 ; 14]$ & $0.6[0 ; 1]$ & $3.5[2 ; 5]$ & $1.4[1 ; 2]$ \\
\hline & 0.1 & $744(71)$ & $23[0 ; 33]$ & $5.1[4 ; 7]$ & $12[9 ; 14]$ & $11[8 ; 14]$ & $0.6[0 ; 1]$ & $3.3[2 ; 5]$ & $1.3[1 ; 2]$ \\
\hline & 0.2 & $716(72)$ & $36[0 ; 60]$ & $4.9[3 ; 7]$ & $12[9 ; 14]$ & $11[6 ; 14]$ & $0.5[0 ; 1]$ & $3.0[1 ; 5]$ & $1.4[1 ; 2]$ \\
\hline
\end{tabular}

predictive interval of observations is represented by the dark blue band. At each time of control, new information is available, it induces adjustment of the prediction of the random effects, the predicted distribution of CD4 and the predicted observations.

For Patient 1, the ORI protocol predicts a cycle of injection at the 2nd, 3rd and 7th visits. However, these cycles are unnecessary as the decrease of CD4 in this patient is very slow. The ACI Protocol correctly predicts that a new cycle will be necessary only at the 5th visit. The ATI protocol predicts a new cycle at a slightly larger time and has the advantage of having only two visits for a control, instead of eight for the other protocols.

Patient 2 has an opposite problem, as the ORI protocol fails to detect that a new cycle was necessary, for example at the first visit, resulting in a long time spent under $500 \mathrm{CD} 4$. In contrast, the ACI and ATI protocols correctly predict that a new cycle is necessary. Again, the number of control visits is reduced in the ATI protocol which yields accurate times of control.

In web supplementary 3, the rate of error, defined as the number of times the decision made is not the optimal, is analyzed. Overall, the rate of time when the algorithm made a decision causing the patient to spend some time under $500 \mathrm{CD} 4$ is extremely low for the ACI and ACIC protocols (between $0.1 \%$ and 6\%). For the ATI and ATIC protocols, it corresponds to the risk taken ( $5 \%$ at the p-quantile of 0.05 ), while this rate was at $47 \%$ for the ORI protocol. The rate of mistake when the algorithm predicts a new cycle for the ACI and ACIC protocols while it could have waited is higher than the ORI protocol, although this has less impact because this decision can be compensated at the next visit. The rate of time when the ATI and ATIC predicted a time of visit too short is around $10 \%$ but this also has less impact because it simply means that the patient has one visit without injections.

Overall, the ATIC protocol with the p-quantile at 0.05 achieves the best balance between all criteria, with a time spent under $500 \mathrm{CD} 4$ among the lowest, and with the number of injections and visits also among the lowest. Compared to the ORI protocol, the ATIC divided the time spend under 500 CD4 by around six, and spared six visits and one injection over a period of two years. However, an analysis done in web supplementary 4 shows that for the patients who have low CD4 at baseline, the ATI protocol with the p-quantile at 0.05 is the best protocol because in these patients we cannot afford to reduce the number of injections per cycle. 
Patient 1
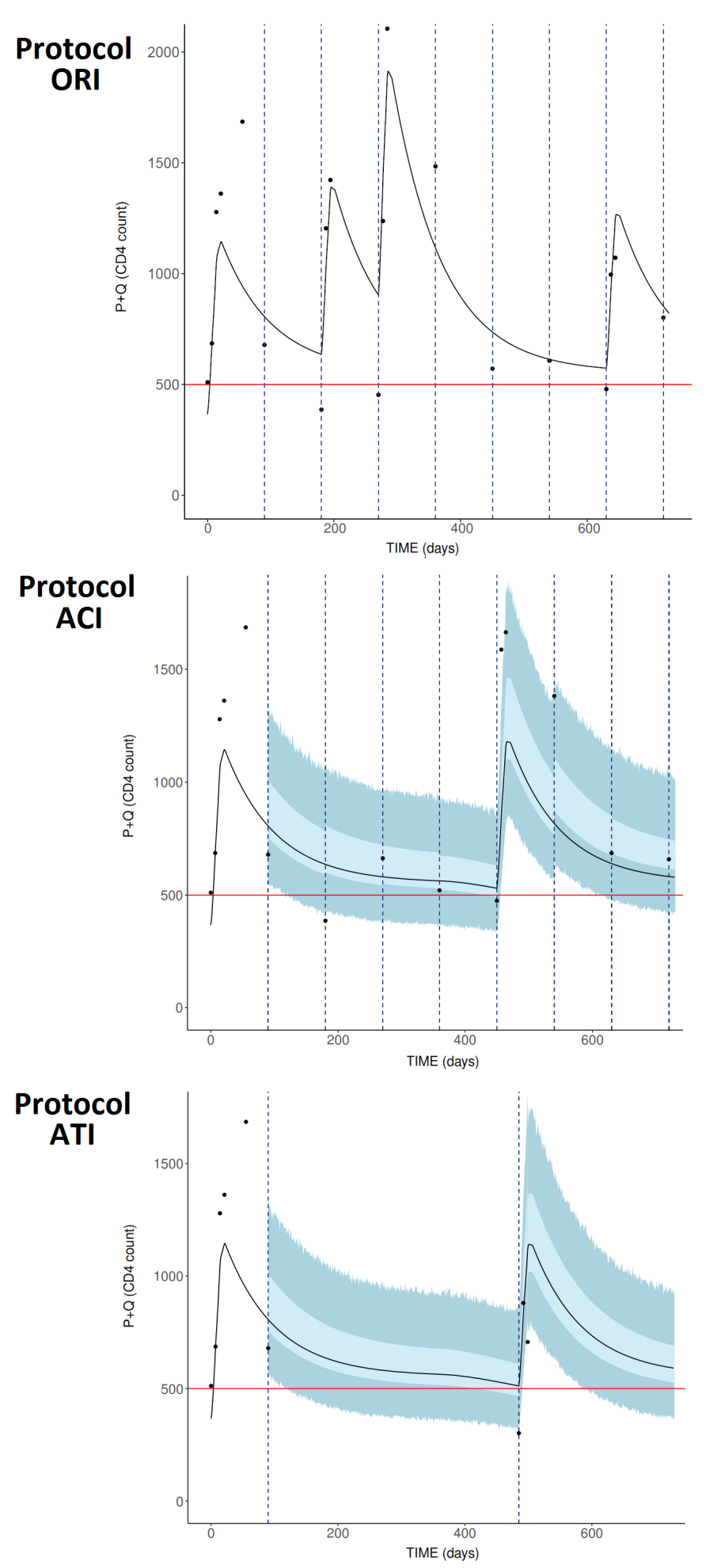

Patient 2
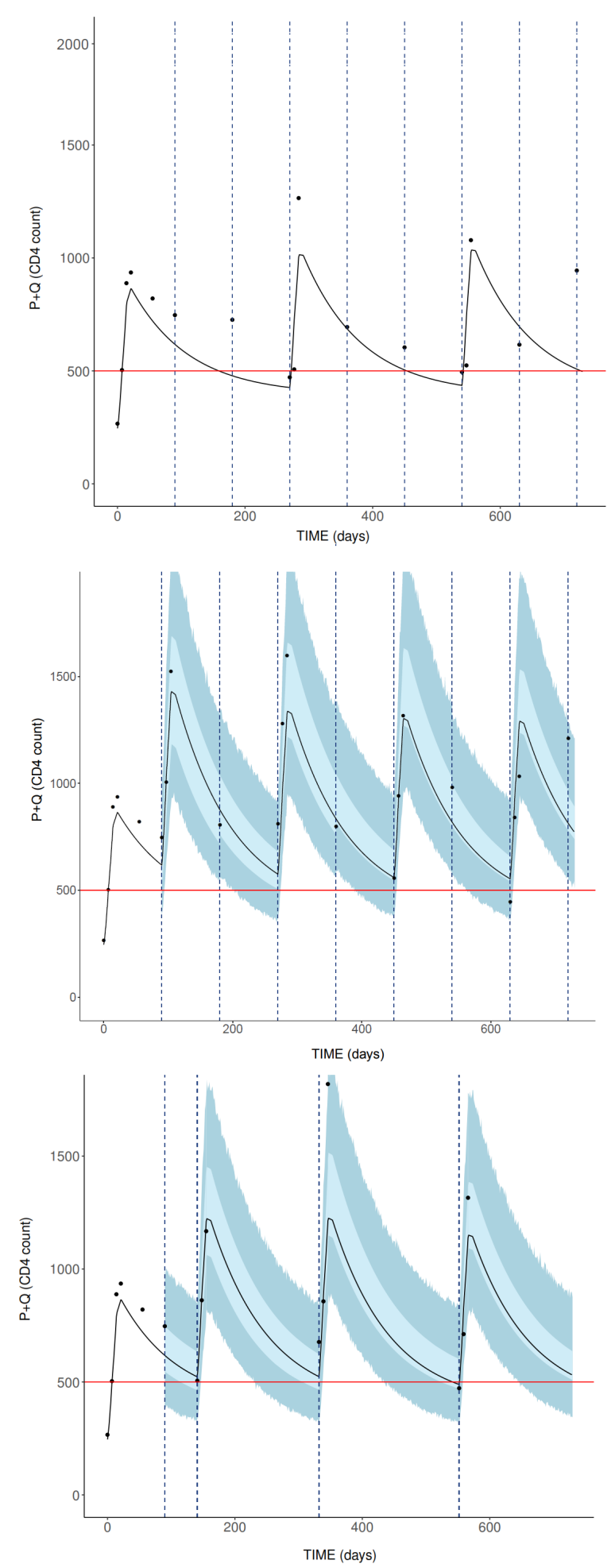

FIGURE 4 Comparison of the three protocols for two random patients. ORI: original protocol, ACI: Adaptive Criterion of Injection Protocol, ATI: Adaptive Time of Injection Protocol. Dots: observations. Black line: simulated trajectory. Horizontal line: limit of 500 CD4. Vertical dashed lines: times of controls. Light band : 95\% credible interval of trajectories. Dark band : $95 \%$ predictive interval of observations. 

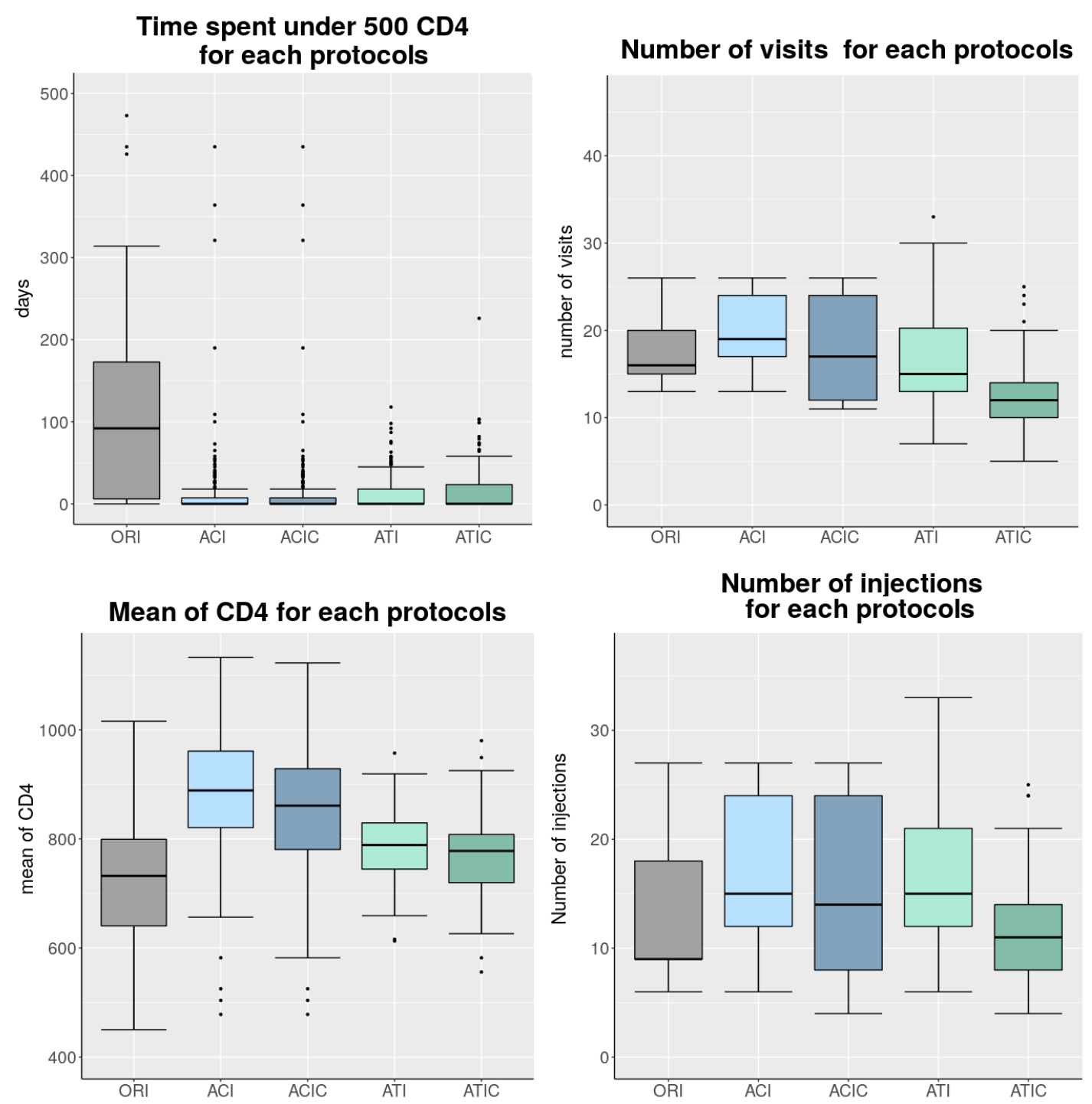

FIGURE 5 Boxplot of time spent under 500 CD4, number of visits, mean number of CD4 and number of injections for each protocol, at the threshold 0.05 .

\section{6 | CONCLUSION}

The very good ability of the mechanistic model to predict CD4 concentrations after a short learning phase to identify individual parameters allows us to embark on optimizing the IL7 administration for each individual patient. Based on this model, we propose adaptive protocols with the aim to optimize a criterion, here the time spent under $500 \mathrm{CD} 4 \mathrm{cells} / \mu \mathrm{L}$, using the minimum number of IL7 injections. The simulation of pseudo-patients showed that the four proposed protocols succeeded in doing so, reducing the time spent under $500 \mathrm{CD} 4 \mathrm{cells} / \mu \mathrm{L}$ with a number of injections similar or lower compared to the original protocol.

Compared to classical approaches that are based on optimal control, our approach presents several advantages. First, the parameters are not considered to be known and the adaptation of the IL7 injections is done while the estimations of the individual parameters and the predictions are improved with new observations. This dynamic approach is also referred as dynamic drug monitoring in the literature ${ }^{25}$. Second, the statistical approach of treatment optimization that we propose is less computationally demanding because we are not looking for an optimal strategy over the space of all potential strategies ${ }^{45}$. Rather, we are optimizing the strategy according to that patient's characteristics, by learning the random effects values as information increases. In our application, this was very relevant because we could take into account the diversity of response of the patients. 
The success of the proposed approach in this application is due to the validity of the predictions that are obtained after a short learning phase for every patient. However, model misspecification could seriously weaken any optimization of the treatment strategy. Here, the model used was clearly the best model over a series of models tested in this context ${ }^{1}$. The stochasticity was mainly due to inter-individuals variability captured through two parameters $(\lambda$ and $\rho$ ), the other parameters are fixed at the value estimated in Jarne et al. ${ }^{1}$ and presented in Table 1 Any additional stochasticity, requiring, for instance, to deal with stochastic differential equations would compromise the feasibility of the approach in a real clinical setting.

The clinical perspective is an evaluation of the adaptive strategy with a standard protocol of injection to confirm the benefit

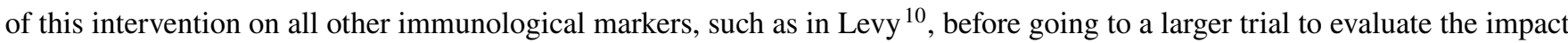
on clinical outcomes. More generally, this work shows how mechanistic model can help increasing the efficiency of therapies in realistic contexts where patients may respond differently to treatments. 


\section{References}

1. Jarne A, Commenges D, Villain L, et al. Modeling $\mathrm{CD}^{+} \mathrm{T}$ cells dynamics in HIV-infected patients receiving repeated cycles of exogenous Interleukin 7. The Annals of Applied Statistics. 2017;11(3):1593-1616.

2. Haase AT. Population biology of HIV-1 infection: viral and CD4+ T cell demographics and dynamics in lymphatic tissues. Annual Review of Immunology. 1999;17(1):625-656.

3. Hammer S.M, Squires K.E, Hughes M.D, et al. A controlled trial of two nucleoside analogues plus indinavir in persons with human immunodeficiency virus infection and CD4 cell counts of 200 per cubic millimeter or less. New England Journal of Medicine. 1997;337(11):725-733.

4. Grabar S, Le Moing V, Goujard C, et al. Clinical outcome of patients with HIV-1 infection according to Immunologic and virologic response after 6 months of highly active antiretroviral therapy. Annals of Internal Medicine. 2000;133(6):401-410.

5. Collaboration Antiretroviral, others . Life expectancy of individuals on combination antiretroviral therapy in high-income countries: a collaborative analysis of 14 cohort studies. Lancet. 2008;372(9635):293.

6. Collaboration Cascade, others . Effective therapy has altered the spectrum of cause-specific mortality following HIV seroconversion. AIDS. 2006;20(5):741-749.

7. Beq S, Delfraissy J-F, Theze J. Interleukin-7 (IL-7): immune function, involvement in the pathogenesis of HIV infection and therapeutic potential. European Cytokine Network. 2004;15(4):279-289.

8. Vassena L, Proschan M, Fauci A.S, Lusso P. Interleukin 7 reduces the levels of spontaneous apoptosis in CD4+ and CD8+ T cells from HIV-1-infected individuals. Proceedings of the National Academy of Sciences. 2007;104(7):2355-2360.

9. Levy Y, Lacabaratz C, Weiss L, et al. Enhanced T cell recovery in HIV-1-infected adults through IL-7 treatment. The Journal of Clinical Investigation. 2009;119(4):997-1007.

10. Levy Y, Sereti I, Tambussi G, et al. Effects of recombinant human interleukin 7 on T-cell recovery and thymic output in HIV-infected patients receiving antiretroviral therapy: results of a phase I/IIa randomized, placebo-controlled, multicenter study. Clinical Infectious Diseases. 2012;55(2):291-300.

11. Sereti I, Dunham R.M, Spritzler J, et al. IL-7 administration drives T cell-cycle entry and expansion in HIV-1 infection. Blood. 2009;113(25):6304-6314.

12. Lewden C, Chêne G, Morlat P, et al. HIV-infected adults with a CD4 cell count greater than 500 cells $/ \mathrm{mm} 3$ on long-term combination antiretroviral therapy reach same mortality rates as the general population. JAIDS Journal of Acquired Immune Deficiency Syndromes. 2007;46(1):72-77.

13. Thiébaut R, Jarne A, Routy J-P, et al. Repeated cycles of recombinant human interleukin 7 in HIV-infected patients with low CD4 T-cell reconstitution on antiretroviral therapy: results of 2 phase II multicenter studies. Clinical Infectious Diseases. 2016;62(9):1178-1185.

14. Perelson A.S, Ribeiro R.M. Modeling the within-host dynamics of HIV infection. BMC biology. 2013;11(1):96.

15. De Boer R.J, Perelson A.S. Quantifying T lymphocyte turnover. Journal of Theoretical Biology. 2013;327:45-87.

16. Lavielle M, Samson A, Karina Fermin A, Mentré F. Maximum Likelihood Estimation of Long-Term HIV Dynamic Models and Antiviral Response. Biometrics. 2011;67(1):250-259.

17. Guedj J, Rong L, Dahari H, Perelson A.S. A perspective on modelling hepatitis C virus infection. Journal of Viral Hepatitis. 2010;17(12):825-833.

18. Ke R, Loverdo C, Qi H, Sun R, Lloyd-Smith J.O. Rational design and adaptive management of combination therapies for Hepatitis C virus infection. PLoS computational biology. 2015;11(6):e1004040. 
19. Kepler G.M, Banks HT, Davidian M, Rosenberg ES. A model for HCMV infection in immunosuppressed patients. Mathematical and Computer Modelling. 2009;49(7-8):1653-1663.

20. Crauste F, Pujo-Menjouet L, Génieys S, Molina C, Gandrillon O. Adding self-renewal in committed erythroid progenitors improves the biological relevance of a mathematical model of erythropoiesis. Journal of Theoretical Biology. 2008;250(2):322-338.

21. Thiébaut R, Drylewicz J, Prague M, et al. Quantifying and Predicting the Effect of Exogenous Interleukin-7 on CD4+ T Cells in HIV-1 Infection. PLoS Computational Biology. 2014;10(5):e1003630.

22. Murphy S.A. Optimal dynamic treatment regimes. Journal of the Royal Statistical Society: Series B (Statistical Methodology). 2003;65(2):331-355.

23. Robins J.M. Optimal structural nested models for optimal sequential decisions. In: :189-326Springer; 2004.

24. Huang X, Ning J, Wahed A.S. Optimization of individualized dynamic treatment regimes for recurrent diseases. Statistics in Medicine. 2014;33(14):2363-2378.

25. Murphy S.A, Lynch K.G, Oslin D, McKay J.R, TenHave T. Developing adaptive treatment strategies in substance abuse research. Drug and Alcohol Dependence. 2007;88:S24-S30.

26. Petersen M.L, Deeks S.G, Laan M.J. Individualized treatment rules: Generating candidate clinical trials. Statistics in Medicine. 2007;26(25):4578-4601.

27. Moodie E.EM, Richardson T.S, Stephens D.A. Demystifying optimal dynamic treatment regimes. Biometrics. 2007;63(2):447-455.

28. Rich B, Moodie E.EM, Stephens D.A. Optimal individualized dosing strategies: A pharmacologic approach to developing dynamic treatment regimens for continuous-valued treatments. Biometrical Journal. 2016;58(3):502-517.

29. Prague M, Commenges D, Gran JM, et al. Dynamic models for estimating the effect of HAART on CD4 in observational studies: Application to the Aquitaine Cohort and the Swiss HIV Cohort Study. Biometrics. 2017;73(1):294-304.

30. Rosenberg Eric S, Davidian Marie, Banks H Thomas. Using mathematical modeling and control to develop structured treatment interruption strategies for HIV infection. Drug \& Alcohol Dependence. 2007;88:S41-S51.

31. Toffoli G, Corona G, Basso B, Boiocchi M. Pharmacokinetic optimisation of treatment with oral etoposide. Clinical Pharmacokinetics. 2004;43(7):441-466.

32. Castiglione F, Piccoli B. Optimal control in a model of dendritic cell transfection cancer immunotherapy. Bulletin of Mathematical Biology. 2006;68(2):255-274.

33. Banks HT, Jang Taesoo, Kwon Hee-Dae. Feedback control of HIV antiviral therapy with long measurement time. Int. J. Pure Appl. Math. 2011;66(4):461-485.

34. Croicu A-M. Short-and Long-Term Optimal Control of a Mathematical Model for HIV Infection of CD4+ T Cells. Bulletin of Mathematical Biology. 2015;77(11):2035-2071.

35. Chakraborty B, Murphy S.A. Dynamic treatment regimes. Annual review of statistics and its application. 2014;1:447-464.

36. Prague M, Commenges D, Drylewicz J, Thiébaut R. Treatment Monitoring of HIV-Infected Patients based on Mechanistic Models. Biometrics. 2012;68(3):902-911.

37. Commenges D, Joly P, Gégout-Petit A, Liquet B. Choice between Semi-parametric Estimators of Markov and Non-Markov Multi-state Models from Coarsened Observations. Scandinavian Journal of Statistics. 2007;34(1):33-52.

38. Prague M, Commenges D, Guedj J, Drylewicz J, Thiébaut R, others . NIMROD: a program for inference via a normal approximation of the posterior in models with random effects based on ordinary differential equations.. Computer Methods and Programs in Biomedicine. 2013;111(2):447-458. 
39. Commenges D, Jacqmin-Gadda H, Proust C, Guedj J. A newton-like algorithm for likelihood maximization: The robustvariance scoring algorithm. arXiv preprint math/0610402. 2006;.

40. Vaart A.W. Asymptotic statistics. Cambridge university press; 2000.

41. Gilks W.R, Best NG, Tan KKC. Adaptive rejection Metropolis sampling within Gibbs sampling. Applied Statistics. $1995 ;: 455-472$.

42. Guedj J, Thiébaut R, Commenges D. Maximum likelihood estimation in dynamical models of HIV. Biometrics. 2007;63(4):1198-1206.

43. Soetaert K, Petzoldt T, Setzer R.W. Solving differential equations in R: package deSolve. Journal of Statistical Software. 2010;33.

44. Hindmarsh A.C. Ordinary differential equation system solver. : Lawrence Livermore National Laboratory; 1992.

45. Pasin C, Dufour F, Villain L, Zhang H, Thiébaut R. Controlling IL-7 injections in HIV-infected patients. arXiv preprint arXiv:1801.06227. 2018;. 
Acknowledgments. We thank the investigators involved in the INSPIRE trials (Jean-Pierre Routy, Irini Sereti, Margaret Fischl, Prudence Ive, Roberto F. Speck, Gianpiero D’Offizi, Salvatore Casari, Sharne Foulkes, Ven Natarajan, Thérèse Croughs, JeanFrançois Delfraissy, Guiseppe Tambussi, Yves Levy, Michael M. Lederman). We thank the MCIA (Mésocentre de Calcul Intensif Aquitain) of the Université de Bordeaux and of the Université de Pau et des Pays de l'Adour for parallel computing. Financial support. Laura Villain gets a PhD grant from Ecole des Hautes Etudes en Santé Publique (EHESP) and Chloé Pasin from Ecole Nationale Supérieure (ENS).

How to cite this article: Villain L., Commenges D., Prague M., Pasin C.,and Thiébaut R. (2018), Adaptive protocols based on predictions from a mechanistic model of the effect of IL7 on CD4 counts, Statistics in Medicine, vol. 